\title{
PCAF ubiquitin ligase activity inhibits Hedgehog/Gli1 signaling in p53-dependent response to genotoxic stress
}

\author{
D Mazzà ${ }^{1,6}$, P Infante ${ }^{2,6}$, V Colicchia ${ }^{1}$, A Greco ${ }^{3}$, R Alfonsi ${ }^{1}$, M Siler $^{1}$, L Antonucci ${ }^{1}$, A Po $^{1}$, E De Smaele ${ }^{4}$, E Ferretti ${ }^{4}$, C Capalbo ${ }^{1}$, \\ D Bellavia ${ }^{1}$, G Canettieri ${ }^{1}$, G Giannini ${ }^{1}$, I Screpanti ${ }^{1,2}$, A Gulino ${ }^{\star, 1,2,5}$ and L Di Marcotullio ${ }^{\star, 1}$
}

The Hedgehog $(\mathrm{Hh})$ signaling regulates tissue development, and its aberrant activation is a leading cause of malignancies, including medulloblastoma (Mb). Hh-dependent tumorigenesis often occurs in synergy with other mechanisms, such as loss of p53, the master regulator of the DNA damage response. To date, little is known about mechanisms connecting DNA-damaging events to morphogen-dependent processes. Here, we show that genotoxic stress triggers a cascade of signals, culminating with inhibition of the activity of Gli1, the final transcriptional effector of $\mathrm{Hh}$ signaling. This inhibition is dependent on the p53-mediated elevation of the acetyltransferase 300/CBP-associated factor (PCAF). Notably, we identify PCAF as a novel E3 ubiquitin ligase of Gli1. Indeed PCAF, but not a mutant with a deletion of its ubiquitination domain, represses Hh signaling in response to DNA damage by promoting Gli1 ubiquitination and its proteasome-dependent degradation. Restoring Gli1 levels rescues the growth arrest and apoptosis effect triggered by genotoxic drugs. Consistently, DNA-damaging agents fail to inhibit Gli1 activity in the absence of either p53 or PCAF. Finally, Mb samples from p53-null mice display low levels of PCAF and upregulation of Gli1 in vivo, suggesting PCAF as potential therapeutic target in Hh-dependent tumors. Together, our data define a mechanism of inactivation of a morphogenic signaling in response to genotoxic stress and unveil a p53/PCAF/Gli1 circuitry centered on PCAF that limits Gli1-enhanced mitogenic and prosurvival response.

Cell Death and Differentiation (2013) 20, 1688-1697; doi:10.1038/cdd.2013.120; published online 6 September 2013

Hedgehog $(\mathrm{Hh})$ is a highly conserved morphogenic pathway that regulates development in several tissues. ${ }^{1}$ Its activation is triggered by the binding of the ligand (Shh, Ihh and Dhh) to the inhibitory receptor Ptch1, which relieves the transmembrane transducer Smoothened (Smo), thus allowing the activation of Gli family of transcription factors (Gli1, 2 and 3). When inappropriately activated (through Ptch1 or SuFu lossof-function or Smo gain-of-function mutations) Hh signaling can lead to tumor formation such as medulloblastoma (Mb). ${ }^{2,3}$ $\mathrm{Mb}$ is the most frequent childhood malignant brain tumor arising from cerebellar neuronal progenitors. Current therapies consist of surgical resection followed by radio- and chemotherapy, which cause however short- and long-term severe adverse effects. ${ }^{4,5}$ This emphasizes the importance to develop new therapeutical approaches to improve prognosis and quality of life of patients. This goal can be achieved by a deeper comprehension of both the molecular events that lead to tumorigenesis and the mechanisms underlying the biological response to the drugs. Although about half of the $\mathrm{Mb}$ tumors display inappropriately high $\mathrm{Hh}$ signaling, mutations of the components of the pathway have been found in only a minority of cases (20-25\%), ${ }^{6}$ suggesting the existence of additional genetic or epigenetic hits. The most powerful effector of the pathway, Gli1, shows strong oncogenic properties as well as mitogenic and prosurvival activity. ${ }^{7-9}$ Gli1 is also a transcriptional target of itself and provides a feedback positive loop, thus reinforcing the strength of the signaling. Therefore, suppression of Gli1 activity represents an effective strategy to restrain $\mathrm{Hh}$ cascade and its oncogenic properties. It has been reported that Gli1 function is finely tuned by posttranslational modifications such as phosphorylation, ubiquitination and acetylation. ${ }^{10-15}$

Recent work suggests that Gli1 function is also controlled by oncosuppressor signaling such as the p53 pathway. ${ }^{16}$ p53 is a well-known transcription factor activated in response to cellular stresses (oncogenic activation, hypoxia and DNA damage) leading to cell cycle arrest or apoptosis. ${ }^{17-19}$ These p53 activities control cellular homeostasis, protecting from tumorigenic events and mediate the suppressive effect of DNA-damaging antitumor drugs.

\footnotetext{
${ }^{1}$ Department of Molecular Medicine, University of Rome La Sapienza, Rome, Italy; ${ }^{2}$ Center for Life NanoScience at LaSapienza, Istituto Italiano di Tecnologia, Italy; ${ }^{3}$ Foundation for Liver Research, Institute of Hepatology, London, UK; ${ }^{4}$ Department of Experimental Medicine, University of Rome La Sapienza, 00161 Rome, Italy and ${ }^{5}$ Neuromed Institute, Pozzilli 86077 , Italy

*Corresponding author: L Di Marcotullio or A Gulino, Department of Molecular Medicine, University of Rome La Sapienza, 291 viale Regina Elena, 00161 Rome, Italy. Tel + 390649255657 or + 3906 4464021; Fax + 3906 49255660; E-mail: lucia.dimarcotullio@ uniroma1.it or alberto.gulino@ uniroma1.it

${ }^{6}$ These authors contributed equally to this work.

Keywords: Hedgehog signaling; ubiquitylation; PCAF; p53; medulloblastoma

Abbreviations: Hh, Hedgehog; Mb, medulloblastoma; PCAF, acetyltransferase p300/CBP-associated factor; Smo, Smoothened; Ub, ubiquitin; Doxo, doxorubicin; Cispl, cisplatin

Received 04.4.13; revised 29.7.13; accepted 30.7.13; Edited by RA Knight; published online 06.9.13
} 
The involvement of p53 in Mb development is indicated by several observations. p53-null genetic background enhances Hh-dependent $\mathrm{Mb}$ formation developed in Ptch1 ${ }^{+/-}$ mice, ${ }^{20,21}$ whereas reduced tumorigenesis is observed in Mdm2-deficient Ptch1 ${ }^{+/-}$mice in which Mdm2-mediated degradation of p53 does not occur. ${ }^{22,23}$ Likewise, individuals with Li-Fraumeni syndrome, harboring germline p53 mutations, ${ }^{24,25}$ are predisposed to the development of Shhtype Mb. ${ }^{26,27}$ Somatic loss-of-function p53 mutations have also been observed in $\sim 14 \%$ of human Shh-group Mb and have been shown to be predictive of shorter survival. ${ }^{26}$ Recently, Shh-Mb has also been reported to display relationship between p53 mutations and chromothripsis, a catastrophic chromosomal rearrangement event associated with more aggressive tumors. ${ }^{28}$ However, the mechanisms through which p53 counteracts $\mathrm{Hh}$ signaling are still poorly investigated.

Here, we show that p53 inhibits Gli1 levels and function in response to DNA damage. This effect is mediated by the induction of PCAF intrinsic E3-ligase activity, leading to Gli1 ubiquitination and proteasome-dependent degradation. This Gli1 inhibition is part of the DNA-damage response in which genotoxic stress attenuates the Gli1 mitogenic and prosurvival properties. Our observations provide a mechanistic explanation of the cooperative role of p53 loss of function with the oncogenic Gli1. The discovery of PCAF as a novel $\mathrm{Hh}$ inhibitor identifies this molecule as a potential therapeutical target in $\mathrm{Mb}$ treatment.

\section{Results}

Genotoxic stress suppresses Hh/Gli signaling. To investigate the role of genotoxic stress on $\mathrm{Hh}$ activity we treated D283 human $\mathrm{Mb}$ cell line with the DNA-damaging agents, doxorubicin or cisplatin. As shown in Figure 1a we found that both drugs suppressed the mRNA levels of Gli1 (a sensitive read out of the pathway) in a dose- and timedependent manner. An increased level of p53 protein was observed as a response to drug-induced DNA damage (Figure 1a, bottom panel). The inhibition of Hh pathway was also confirmed by reduction of other $\mathrm{Hh}$ target genes (cyclin D2, Hip1, Bcl2 and Bmi1) (Figure 1b). The same effect on Gli1 mRNA levels was observed in MEF Ptch $1^{-1-}$, in which the Hh pathway is constitutively activated as a consequence of the loss of the inhibitory receptor Ptch1 (Supplementary Figure 1). Doxorubicin or cisplatin also suppressed $\mathrm{Hh}$ signaling in NIH 3T3 Shh light II cells, stably incorporating a Gli1-responsive reporter, ${ }^{29}$ as indicated by inhibition of luciferase activity in cells treated with the Smo agonist SAG (Figure 1c). These findings suggest that DNA damage suppresses Hedgehog signaling under basal or activated conditions. Consistently with the drug-induced $\mathrm{Hh}$ inhibition, we observed the downregulation of Gli1 protein levels in both D283 and MEF Ptch1 ${ }^{-1}$ cells (Figure 1d).

Our observations suggest that these chemotherapeutic drugs display antitumor properties in $\mathrm{Hh}$-dependent $\mathrm{Mb}$ through the inhibition of Hh/Gli signaling.
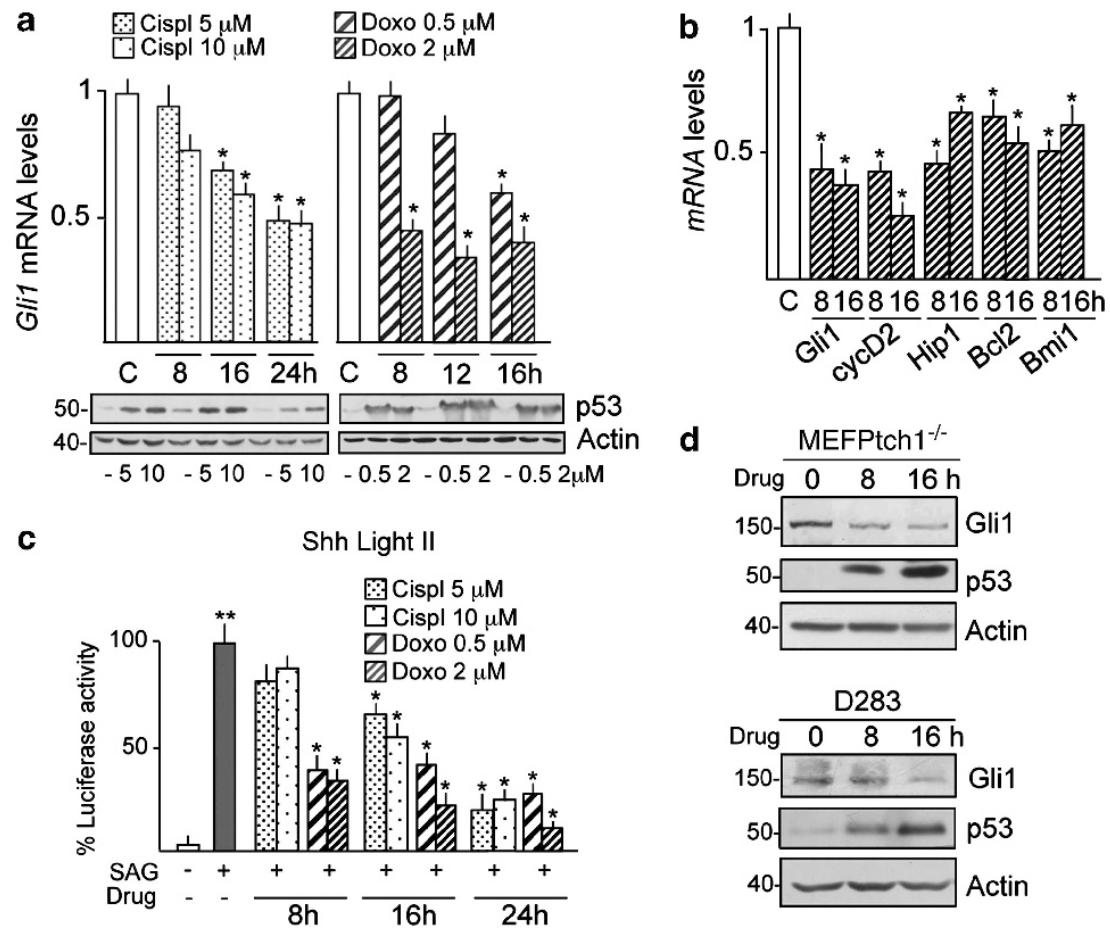

Figure 1 Genotoxic stress suppresses Hh signaling. (a) D283 Mb cells were treated with cisplatin (Cispl; left), or doxorubicin (Doxo; right), in a dose- and time-dependent manner, as indicated in the figure. Levels of Gli1 mRNA were analyzed by quantitative PCR (mean arbitrary units \pm S.D.). ${ }^{*} P<0.05$, drugs versus control. Representative immunoblotting analysis of p53 protein levels in untreated and treated cells (bottom). (b) RNAs from D283 cells exposed to doxorubicin $(2 \mu \mathrm{M})$ for the indicated times were analyzed by quantitative PCR, as described in (a). ${ }^{*} P<0.05$, doxorubicin versus control. (c) Shh Light Il cells were treated for $48 \mathrm{~h}$ with $S A G$ or DMSO and with cisplatin or doxorubicin for the indicated times and luciferase activity was tested. ${ }^{*} P<0.02$, SAG versus control; ${ }^{*} P<0.05, S A G+$ drugs versus $S A G$. (d) Immunoblotting analysis of Gli1 and p53 protein levels in D283 and MEF Ptch1 ${ }^{-1-}$ cells untreated and treated with $2 \mu \mathrm{M}$ doxorubicin. In all experiments MW of indicated protein are in $\mathrm{kDa}$ 
Gli1 suppression in response to DNA damage requires p53. To understand how genotoxic stress represses Gli1 levels, we investigated the involvement of p53, a tumor suppressor and master regulator of the DNA damage response that has been described to also inhibit $\mathrm{Hh} / \mathrm{Gli1}$ signaling. ${ }^{16}$ To this end, we analyzed Gli1 expression in p53depleted cells after doxorubicin treatment. The experiment was performed in D283 cells transduced with lentivirus, allowing stable expression of either control or p53 short hairpin RNAs (shRNAs). Depletion of endogenous p53 abrogated the modulation of Gli1 induced by DNA damage (Figure 2a). Also, we did not observe Gli1 downregulation (at both mRNA and protein levels) in Daoy Mb cells carrying a p53 loss-of-function mutation, ${ }^{30}$ although the doxorubicininduced DNA damage was confirmed by observing elevated expression of $\gamma \mathrm{H} 2 \mathrm{AX}$ (Figure $2 \mathrm{~b}$ ). Similar results were obtained in p53-null Saos-2 cells compared with p53 wild type U2OS or D341 Mb cells ${ }^{30}$ (Supplementary Figure 2). Overall, these findings suggest that p53 is required for the suppression of Gli1 in response to DNA damage.

Genotoxic stress induces PCAF in a p53-dependent manner. A common mechanism mediating p53-dependent response to genotoxic stress requires the activation of a

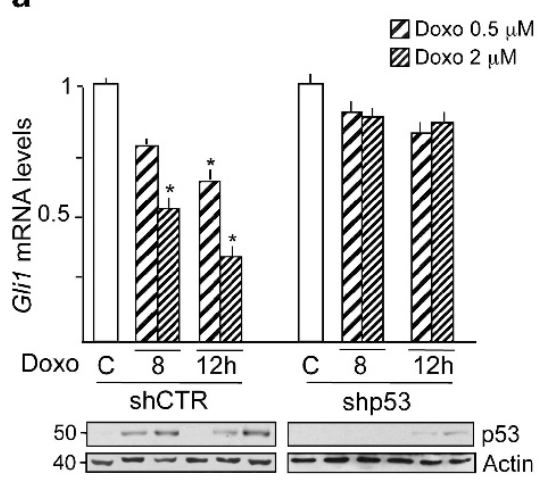

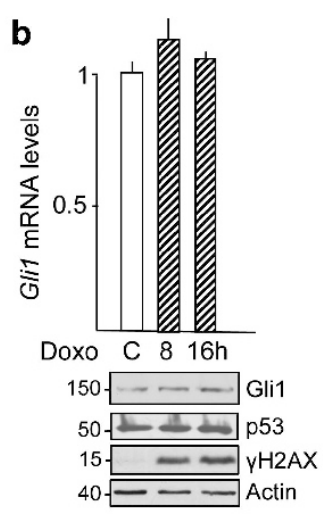

d Cisplatin
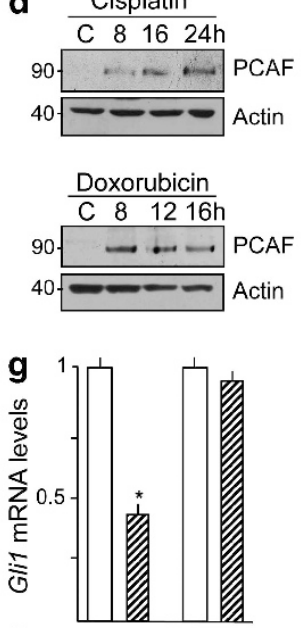

Doxo

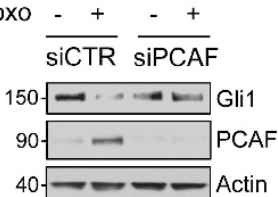

Figure 2 Gli1 suppression in response to DNA damage requires p53 and PCAF. (a) Stable D283-derived cell line expressing either a control shRNA (shCTR) or an shRNA directed against p53 (shp53) were treated with doxorubicin (Doxo) in a dose- and time-dependent manner, as indicated in the figure. Levels of Gli1 mRNA were analyzed by quantitative PCR (mean arbitrary units \pm S.D., from three independent experiments). ${ }^{*} P<0.05$, doxorubicin versus control. Representative immunoblotting analysis of p53 protein levels in untreated and treated cells (bottom). (b) Daoy cells were treated with $2 \mu \mathrm{M}$ doxorubicin for the indicated times. Levels of Gli1 mRNA were analyzed by quantitative PCR. Representative immunoblotting analysis of Gli1, $\gamma \mathrm{H} 2 \mathrm{AX}$ and p53 protein levels in untreated and treated cells (bottom). (c) D283 cells were treated with doxorubicin or cisplatin in a dose- and time-dependent manner, as indicated in the figure. Levels of $p 300$ and $P C A F$ mRNA were analyzed by quantitative PCR. ${ }^{*} P<0.05$, drugs versus control. (d) Representative immunoblotting analysis of PCAF protein levels after doxorubicin or cisplatin treatment. (e) D283 (left) or Daoy (right) cells were treated with $2 \mu \mathrm{M}$ doxorubicin for the indicated times. Levels of $P C A F$ mRNA were analyzed by quantitative PCR. ${ }^{*} P<0.05$, doxorubicin versus control. Representative immunoblotting analysis of PCAF, p53 and $\gamma \mathrm{H} 2 \mathrm{AX}$ protein levels in untreated and treated cells (bottom). (f) Levels of PCAF mRNA analyzed by quantitative PCR in D283 p53depleted cells as described in (a). ${ }^{*} P<0.05$, doxorubicin versus control. (g) Gli1 mRNA levels and immunoblotting analysis of Gli1 and PCAF protein in D283 cells transfected with siRNA specific for PCAF or a non-specific control and treated with $2 \mu \mathrm{M}$ doxorubicin for $12 \mathrm{~h}$. In all experiments MW of indicated protein are in $\mathrm{kDa}$ 
acetyltransferases (HATs) p300 and $\mathrm{PCAF}^{17}$ and the acetylation of different substrates. We wondered whether these coactivators were involved in p53-mediated Gli1 inhibition. We observed that doxorubicin or cisplatin significantly upregulated mRNA and protein levels of PCAF, whereas no effect was observed on p300 (Figures 2c and d). PCAF upregulation was p53-dependent, like the previously shown DNA damage induced downregulation of Gli1 (Figures 2a and f). PCAF modulation was observed only in p53-proficient cells (D283 and U2OS, Figure $2 e$ and Supplementary Figure 2), but not in Daoy (p53 lossof-function), Saos-2 (p53-null) and in D283 p53-depleted cells (Figures $2 \mathrm{e}$ and f, Supplementary Figure 2). PCAF has been reported to be a transcriptional target of $p 53 .^{31}$ To determine whether p53 is recruited to PCAF promoter in our cellular context, we performed quantitative chromatin immunoprecipitation assay (ChIP) in D283 cells treated with doxorubicin. ChIP experiments showed that p53 was recruited to the PCAF promoter in response to DNA damage in association with an increase of acetylated histone $\mathrm{H} 3$ (Supplementary Figure 3), indicating that p53 binds to transcriptionally active chromatin. Interestingly, p53-dependent modulation of Gli1 and PCAF exhibited an inverse correlation. This observation prompted us to investigate whether PCAF was required for Gli1 downregulation in response to DNA damage. Prevention of PCAF accumulation by small interference RNA (siRNA) antagonized the genotoxic drug-induced suppression of Gli1 mRNA and protein in
D283 cells (Figure $2 \mathrm{~g}$ ). Together, these results demonstrate that p53-induced upregulation of PCAF is required for the inhibition of Gli1 in response to DNA damage.

PCAF acetyltransferase activity is dispensable for Gli1 suppression. To deepen our observation, we tested the ability of PCAF to inhibit Gli1 function. PCAF overexpression decreased Gli1-induced luciferase activity driven by a Gli-responsive construct to a similar extent as p300 (Figure $3 a$ ) that we previously described to acetylate and suppress Gli1 transcriptional activity. ${ }^{32}$ Surprisingly, a mutant of PCAF deleted of the acetyltransferase domain (PCAFA HAT) kept its inhibitory effect (Figure 3a). Accordingly, we observed that Gli1 was not acetylated by PCAF in vivo (Figure 3b). These results demonstrate that PCAF acetyltransferase activity is dispensable for Gli1 inhibition.

PCAF ubiquitinates and degrades Gli1. To determine whether the effect of PCAF on Gli1 requires their physical interaction, we carried out co-immunoprecipitation and GST pulldown assays. Both in vivo (Figures $3 \mathrm{c}$ and $\mathrm{d}$ ) and in vitro (Figure 3e) studies revealed that PCAF and Gli1 form a complex. In light of this result and having excluded the involvement of PCAF acetyltransferase activity in the inhibition of Gli1, we investigated how PCAF could impair Gli1 function.

In addition to acetyltransferase activity, PCAF has been recently described to possess intrinsic ubiquitin ligase
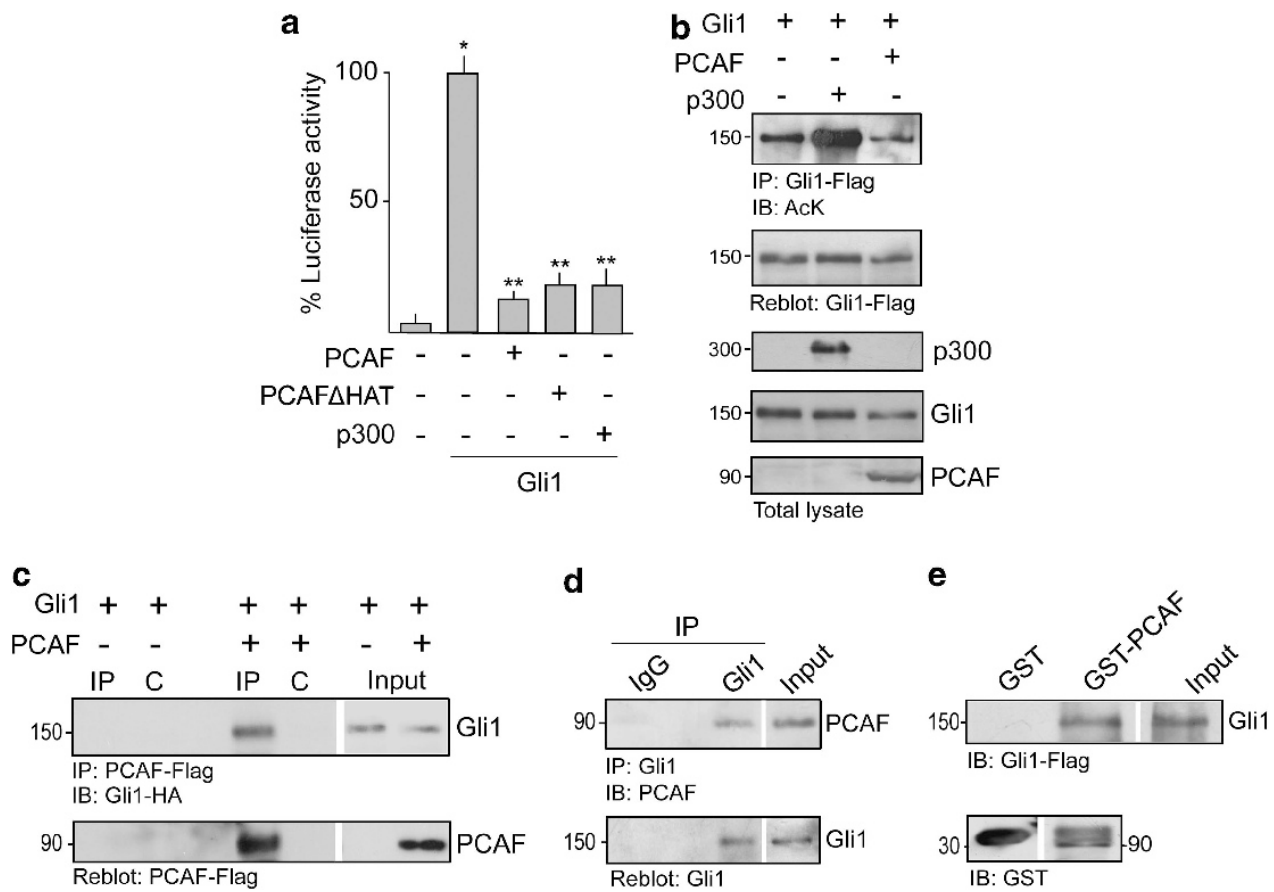

Figure 3 PCAF HAT activity is dispensable for Gli1 suppression. (a) Luciferase assay performed in HEK293T cells transfected with Gli1 and $12 \times$ Gli Luc reporter, alone or in combination with PCAF or PCAF $\triangle$ HAT or p300. ${ }^{*} P<0.02$, Gli1 versus empty; ${ }^{* *} P<0.02$ Gli1 + PCAF or Gli1 + PCAF $\Delta$ HAT or Gli1 + p300 versus Gli1. (b) HEK293T cells were transfected with Gli1 alone or in combination with PCAF or p300. After immunoprecipitation with Flag antibody, acetylated Gli1 was detected by anti-acetylated lysine antibody (AcK). The blot was reprobed with Flag antibody. Lower panels show p300, Gli1 and PCAF protein levels in total cell lysate. (c) HEK293T cells were transfected with Gli1 in the absence or presence of PCAF, immunoprecipitated with anti-Flag agarose beads and immunoblotted with HA antibody. As a negative control, beads were pre-blocked with Flag peptide $(0.1 \mathrm{mg} / \mathrm{ml})$. (d) Cell lysates from HEK293T cells were immunoprecipitated with rabbit-Gli1 antibody or control rabbit-antisera (lgG) and immunoblotted with Gli1 and PCAF antibodies. (e) GST-PCAF was bound to glutathione-sepharose beads and used for in vitro pulldown assay. Gli1 was in vitro-translated and then incubated with free GST control or GST-PCAF. Protein complexes were detected by immunoblot assay. In all experiments MW of indicated protein are in kDa 
function leading to Mdm2 ubiquitination and degradation. ${ }^{33}$ Therefore, we investigated the ability of PCAF to ubiquitinate Gli1. Immunoprecipitation experiments showed that PCAF induces the ubiquitination of Gli1 in a dose-dependent manner (Figure 4a, left). Similar results were observed in the presence of a PCAF $\triangle$ HAT mutant (Figure $4 a$, middle), but not upon overexpression of the acetyltransferase p300 (Figure $4 \mathrm{a}$, right), also described to harbor ubiquitin ligase properties. ${ }^{34}$ PCAF most likely affected poly-ubiquitination, as demonstrated by its failure to promote Gli1 ubiquitination in presence of ubiquitin[mono]substrate (Supplementary Figure 4a). Notably, PCAF deleted of its ubiquitination domain (PCAF $\Delta U b)^{33}$ was ineffective (Figure $4 \mathrm{~b}$ and Supplementary Figure $4 \mathrm{~b}$ ). These observations demonstrate that PCAF is a novel E3-ubiquitin ligase for Gli1.
To link PCAF E3-ligase activity to the impairment of Gli1 function, we assessed whether PCAF also induces Gli1 degradation. In agreement with previous data pointing to the involvement of the proteasome system in the processing of ubiquitinated Gli1 protein, ${ }^{35,36}$ cells treated with the proteasome-inhibitor MG132 displayed significant accumulation of ubiquitinated Gli1 (Figure 4c). This observation suggests that Gli1 is degraded following PCAF-mediated ubiquitination.

Steady-state levels of Gli1 protein were decreased following transfection of increasing amounts of PCAF, but not of PCAFAUb mutant (Figure 4d). Further, siRNA-mediated PCAF depletion increased the half-life of Gli1 protein, supporting the role of PCAF to promote Gli1 proteolysis (Figure 4e).

To investigate whether PCAF-induced ubiquitination affects distinct Gli1 functional domains, we performed ubiquitination

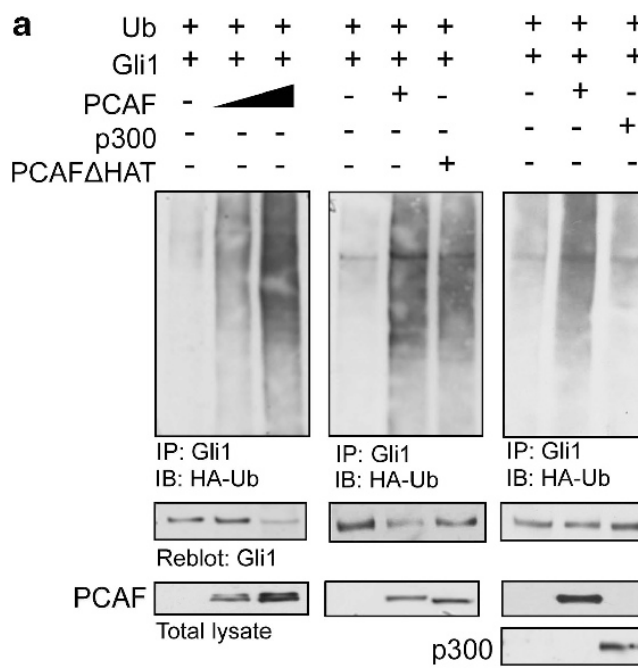

b

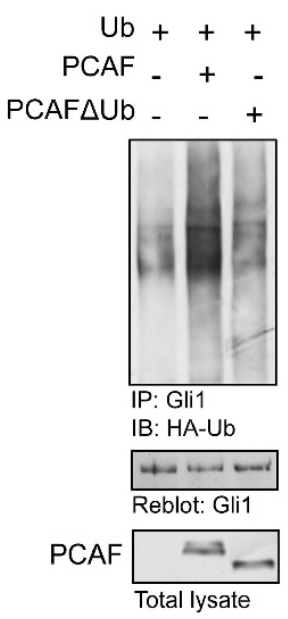

C

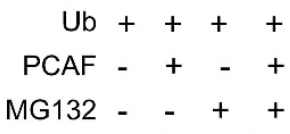

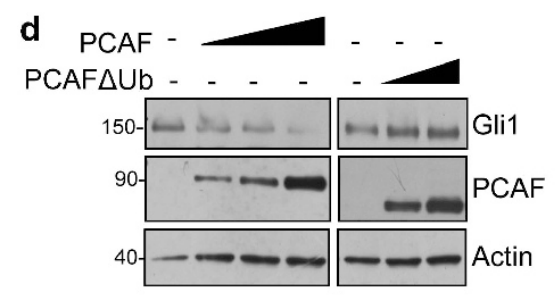

e
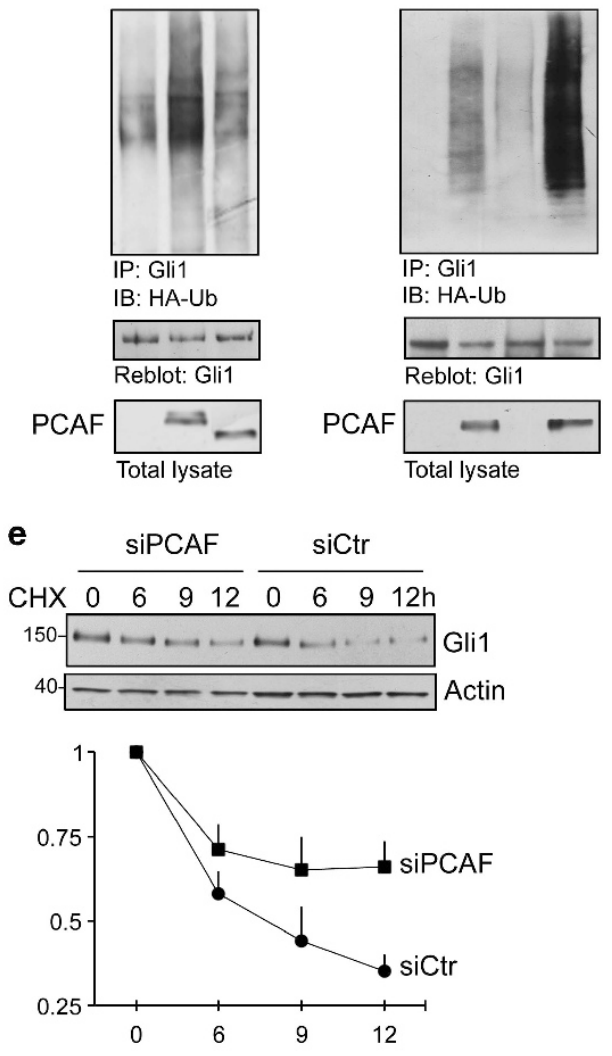

Figure 4 PCAF ubiquitinates and degrades Gli1. (a) HEK293T cells were co-transfected with HA-Ubiquitin and Gli1 in the absence or presence of increasing amounts of PCAF or PCAF $\triangle \mathrm{HAT}$ or $\mathrm{p} 300$. Cellular lysates were immunoprecipitated with Gli1 antibody, followed by western blot with HA antibody to detect coniugated HA-Ub. The blot was reprobed with Gli1 antibody. PCAF and p300 protein levels in total cell lysate (bottom). (b) HEK293T cells were transfected with HA-Ub in the presence or absence of PCAF or PCAF $\triangle \mathrm{Ub}$ mutant. Endogenous Gli1 was immunoprecipitated with rabbit-Gli1antibody and ubiquitinated forms revealed as described in (a). PCAF protein levels in total cell lysate (bottom). (c) HEK293T expressing HA-Ub in the presence or absence of PCAF were treated with the proteasome inhibitor MG132 (50 $\mu \mathrm{g} / \mathrm{ml})$ for $6 \mathrm{~h}$ prior of lysis. Immunoprecipitation of endogenous Gli1 was performed as in (a). PCAF protein levels in total cell lysate (bottom). (d and e) Degradation of transfected Flag-Gli1 in HEK293T in the presence of increasing amount of Flag-PCAF or Flag-PCAF $\triangle \mathrm{Ub}$ (d) or after treatment with $30 \mu \mathrm{g} / \mathrm{ml}$ cycloheximide (CHX) in the presence of control or PCAF siRNAs for the indicated time points (e). The graphs indicate densitometric analysis of three independent experiments (mean arbitrary units \pm S.D.). Data are shown as a ratio of Gli1/Actin normalized to the amount of Gli1 in absence of overexpressed PCAF (d) or at the beginning of CHX treatment (e). In all experiments, MW of indicated proteins are in $\mathrm{kDa}$ 
and transactivation assays on either $\mathrm{N}$-terminal (aa 1-424) or C-terminal (aa 424-1106) regions. To this end we tested two types of fusion proteins: $\mathrm{N}$ terminus of Gli1 (containing the DNA-binding domain) fused to the VP16 transactivation domain (Gli1N-VP16) and C terminus of Gli1 (containing the transactivating domain) fused to the Gal4 DNA-binding domain (Gal4-Gli1C) (Figure 5a). Gli1 C terminus (Gal4-Gli1C), but not its N terminus (Gli1N-VP16), was ubiquitinated by both PCAF and PCAF $\triangle$ HAT (Figures 5b and c). Accordingly, while PCAF significantly decreased the activity of Gal4-Gli1C fusion protein on a Gal4-luciferase reporter, the activity of transfected Gli1NVP16 fusion protein was not affected (Figure 5d). These findings indicate that the PCAF-responsive site of Gli1 is functionally located on the $\mathrm{C}$ terminus. Docking sites for others Gli1 E3 ubiquitin ligases ( $\beta$ TrCP, $D_{C}$ and Itch, $\left.D_{l}\right)$ (Figure $\left.5 a\right)$ have been described to reside in this region. ${ }^{11,36}$ However, we did not observe changes in the PCAF-induced ubiquitination levels of Gli1 mutants lacking $\beta \operatorname{TrCP}$ (Gli1 $\Delta \mathrm{Dc}$ ) or Itch (Gli1) degrons (Supplementary Figures $5 \mathrm{a}$ and $\mathrm{b}$ ).

Gli1 ubiquitination in response to DNA damage requires PCAF and p53. The ability of PCAF to impair Gli1 stability suggests that DNA damage would suppress Gli1 function by promoting its ubiquitination. Indeed, doxorubicin increased the ubiquitination of endogenous Gli1 in D283 cells, in association with increased PCAF levels (Figure 6a). This event was abrogated when PCAF expression was depleted by siRNA-knockdown (Figure 6a). As a result of the ability of p53 to activate PCAF transcription in response to DNA damage, Gli1 ubiquitination and PCAF induction by doxorubicin were significantly reduced in D283 cells transduced with an shp53 (Figure 6b). Overall, these findings suggest that the inhibition of $\mathrm{Hh}$ signaling by DNA damage is due to Gli1 ubiquitination by p53-induced PCAF expression.

p53-mediated PCAF activation prevents the growth and survival promoting activity of Gli1. To determine whether the above-described p53/PCAF/Gli1 pathway works in vivo, we analyzed Gli1 and PCAF levels in Mb tumors isolated from Ptch $1^{+/-}$or Ptch $1^{+I-} ; \mathrm{p} 53^{-I-}$ mice. Remarkably, PCAF levels were significantly downregulated, whereas Gli1 levels were upregulated in Ptch $1^{+/-} ; \mathrm{p} 53^{-/-}$compared with Ptch1 ${ }^{+/}$Mb (Figure 7a). This is consistent with the model whereby p53 suppresses Gli1 through activation of PCAF.

To address the involvement of PCAF/Gli1 pathway in restraining tumor cell growth, we studied D283 cell proliferation and survival in response to doxorubicin treatment. As evaluated by BrdU incorporation assay, the drug-induced growth arrest was rescued by exogenous Gli1 (Figure 7b). Gli1 enhanced levels also abolished the doxorubicin-induced

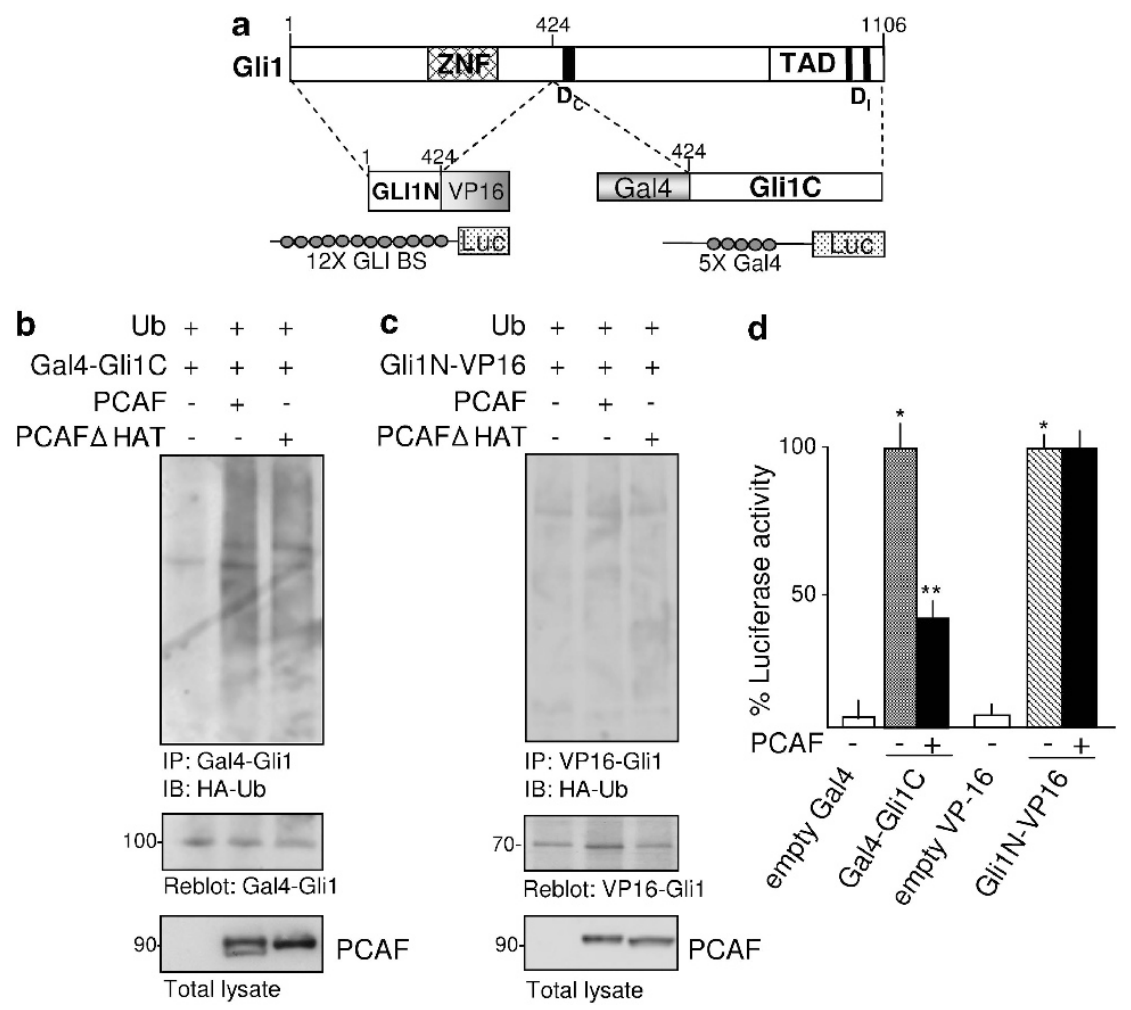

Figure 5 PCAF-responsive site of Gli1 is located at the C terminus. (a) Schematic representation of Gli1 and subdomains used to generate the Gli1N-terminal VP16 and Gal4 C-terminal fusion proteins and reporters. Gli1 (2-424 aa) fragment was fused to VP16 transactivation domain (Gli1N-VP16); Gli1 (424-1106 aa) fragment was fused to the Gal4 DNA-binding domain (Gal4-Gli1C). ZNF: DNA-binding domain; TAD: trans-activation domain; $D_{C}$ : $\beta$ TrCP-binding site; $D_{1}$ : Itch-binding site. (b and c) Gal4-Gli1C (b) or Gli1N-VP16 (c) mutants were co-transfected in HEK293T cells with HA-Ub in the presence or absence of PCAF or PCAF $\triangle$ HAT. Cellular lysates were immunoprecipitated with Gal4 (b) or VP16 (c) antibodies, followed by western blot analysis using an HA antibody to detect conjugated HA-Ub. The blot was reprobed with the indicated antibodies. PCAF protein levels in total cell lysates (bottom panel). (d) A luciferase assay was performed in HEK293T cells transfected with: Gli1N-VP16 and 12XGli Luc reporter, alone or in combination with PCAF or with Gal4-Gli1C and Gal4-responsive luciferase reporter, in the absence or presence of PCAF. ${ }^{*} P<0.02$, Gal4-Gli1C versus empty Gal4 or Gli1N-VP16 versus empty VP16; ${ }^{* *} P<0.02$, Gal4-Gli1C + PCAF versus Gal4-Gli1C. In all experiments, MW of indicated proteins are in kDa 

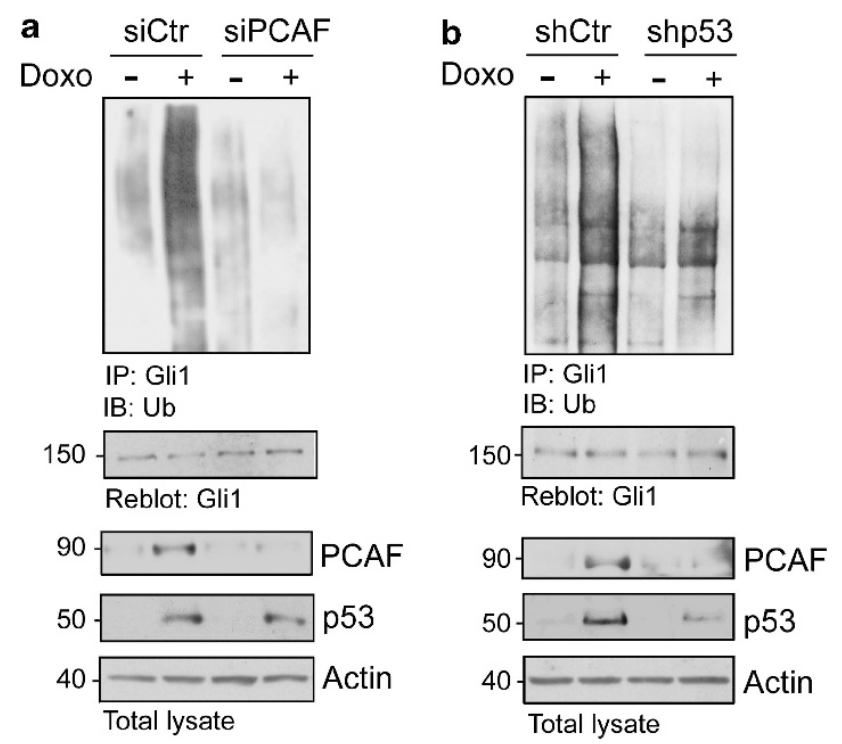

Figure 6 PCAF ubiquitinates Gli1 in response to genotoxic stress via p53. (a and b) D283 cells were transfected with siRNA specific for PCAF or a non-specific control (a) or transduced with shp53 or a non-specific control (b) and treated with $2 \mu \mathrm{M}$ doxorubicin for $12 \mathrm{~h}$. Cellular lysates were immunoprecipitated with rabbit-Gli1 antibody, followed by immunoblotting with mouse-Ubiquitin (Ub) antibody. The blots were reprobed with mouse-Gli1 antibody. PCAF and p53 protein levels in total cell lysate (bottom). In all experiments MW of indicated protein are in $\mathrm{kDa}$

apoptosis, as assessed by the expression of the PARPcleaved form and the presence of apoptotic nuclei (Figure 7c). These results suggest that Gli1 impairment is involved in both growth arrest and apoptosis in response to DNA damage. Consistently, drug-dependent effect requires PCAF, as siRNA-mediated PCAF depletion abrogated Mb cells sensitivity to doxorubicin-induced growth arrest and cell death (Figures 7d and e).

PCAF may increase p53 levels by inducing Mdm2 degradation. ${ }^{33}$ To rule out the involvement of this mechanism, we analyzed the effect of PCAF on Mb cell growth in Daoy cells harboring a defective p53 mutation. ${ }^{30}$ Exogenous PCAF, but not PCAF $\triangle U b$, a mutant unable to induce Gli1 degradation, decreased cell growth in this cell line as evaluated by BrdU incorporation assay (Figure 7f). Importantly, overexpressed PCAF inhibited cell growth to the same extent as Gli1 siRNA-mediated knockdown and combined PCAF overexpression in Gli1 knockdown did not inhibit cell growth to greater extent. These results suggest that these proteins are in the same pathway for inhibition of cell proliferation (Figure 7f). Colony-formation assays confirmed the role of PCAF to modulate Mb cell growth by inhibition of Gli1. As expected, Gli1 overexpression or depletion promoted or inhibited, respectively, Mb colony-formation ability (Figure $7 \mathrm{~g}$ and Supplementary Figure 6a). Accordingly, ectopic expression of PCAF, but not PCAF $\Delta U b$, caused a significant decrease in the number of colonies compared with cells transfected with the empty vector (Figure $7 \mathrm{~g}$ ). The effect of Gli1, PCAF and PCAF $\triangle U$ b on Mb cell proliferation was also supported by an analysis of cumulative cell number over time (Figure 7h and Supplementary Figure 6b). Together, our findings suggest that degradation of Gli1 by PCAF has an important role in restraining the Hh-dependent $\mathrm{Mb}$ growth in response to genotoxic stress.

\section{Discussion}

Aberration of the Hedgehog pathway leads to malignancies, including medulloblastoma. Hh-dependent tumorigenesis is favored by the loss of p53, but the mechanisms connecting DNA-damage response to morphogen-dependent control of cancer are still unclear. In this work we identify a novel molecular mechanism (Figure 8), whereby drug-induced DNA damage triggers a cascade of events that results in $\mathrm{Hh} / \mathrm{Gli}$ inhibition through the activation of the PCAF/p53 axis. We observed that genotoxic stress downregulates the mRNA and protein levels of Gli1 and blunts its function. We demonstrated that these effects are strictly dependent on the presence of a functionally active p53 that in response to DNA damage triggers the transcription of PCAF and promotes its accumulation. The intrinsic E3-ligase activity of PCAF is then responsible for the ubiquitin-dependent degradation of Gli1, thus restraining its mitogenic and prosurvival activity. Interestingly, these observations are consistent with the enhanced growth arrest and cell death induced by the Gli1 antagonist GANT61. ${ }^{37,38}$ Targeting the Hh pathway has a crucial role for the therapy of Hh-dependent tumors (e.g. Mb). ${ }^{39}$ Inhibition of Smo protein is currently exploited by several small molecules in clinical trials. However, acquired resistance to these drugs has been reported, as a consequence of subsequent amplification of Gli2 or further mutations of Smo or activation of overcoming oncogenic pathways. ${ }^{40,41}$ A still unresolved issue is represented by the targeting of Gli1, whose small molecule inhibitors are at a very early stage of development. Therefore, a promising therapeutic strategy would be targeting the mechanisms that lead to inactivation (i.e. degradation) of Gli1. The identification of PCAF as a key enzyme to inhibit Gli1 would allow the use of p53-activating drugs (e.g. DNA-damaging drugs or the inhibitors of Mdm2/ p53 interaction, Nutlin-3 or RITA) in combination therapies of Hh-dependent tumors or to control the disease recurrence due to acquired anti-Smo drug-resistance. Remarkably, Nutlin-3 and RITA are currently tested as promising anticancer treatments. ${ }^{42}$ Our observations might explain the ability of Nutlin-3 treatment to suppress Mb, which in most cases display high Mdm2 levels. ${ }^{43,44}$

In conclusion, we have described a p53/PCAF/Gli1 circuitry centered on PCAF, whose coordinated p53 acetylating and Gli1 ubiquitin/proteolytic functions are candidates as therapeutic targets, thus preventing the Hh-mediated tumorigenic process. Likewise, Hh/Gli antagonists are predicted to sensitize cancer cells to the tumor-suppressive effect of p53-enhancing drugs due to the role of both p53 and $\mathrm{Hh}$ pathways in cell growth and apoptosis. Conversely, aberrant Hh activation together with loss of PCAF-mediated restraining signals (e.g. loss of p53) would enhance tumorigenesis, a process that might be counteracted by PCAF-activating drugs.

\section{Materials and Methods}

Cell culture, tranfection and reagents. HEK293T, MEF Ptch $1^{-1-}$, Shh light II, U2OS and Saos-2 were cultured in DMEM plus 10\% FBS; D283 and Daoy 

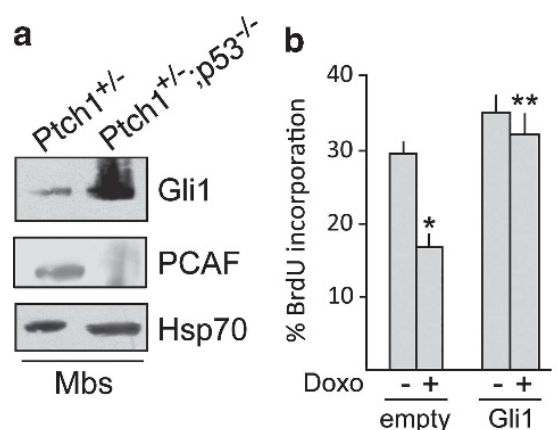

C $\square$ CPARP+

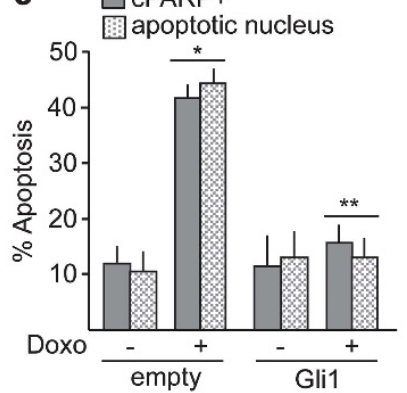

d

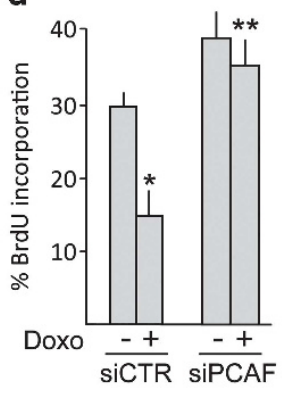

e

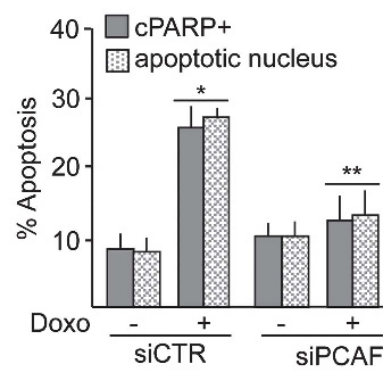

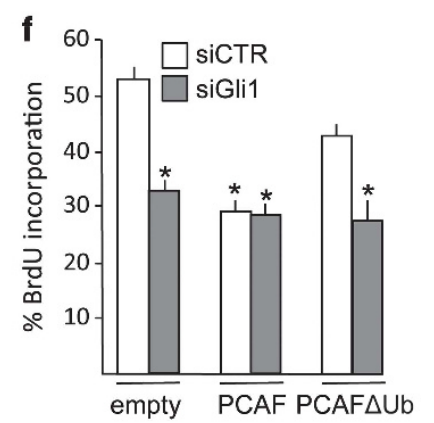
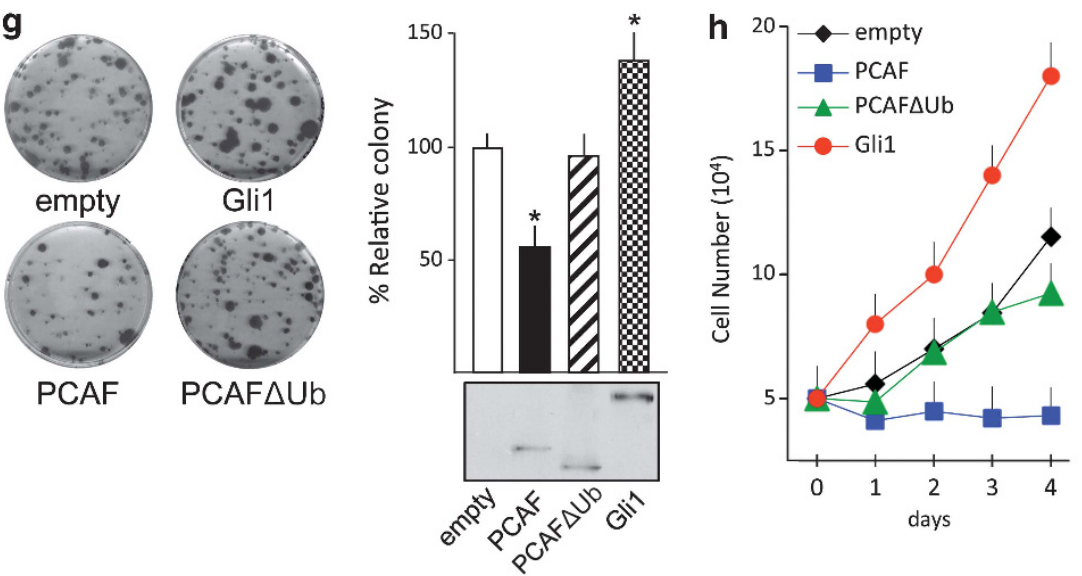

Figure 7 PCAF prevents the mitogenic and prosurvival activity of Gli1 in response to genotoxic stress. (a) Protein levels of Gli1 and PCAF in Mb tumor samples from Ptch $1^{+I-}$ or Ptch1 $1^{+1-} ; \mathrm{p} 53^{-1-}$ mouse model. (b) D283 medulloblastoma cells were transfected for $24 \mathrm{~h}$ with Gli1 or control vector and then treated with $2 \mu \mathrm{M}$ doxorubicin. Percent of BrdU incorporation was assessed in the population of transfected cells to monitor cell proliferation. ${ }^{*} P<0.05$, doxo versus control; ${ }^{* *} P<0.05 \mathrm{Gli} 1+$ doxo versus empty + doxo. (c) D283 cells were transfected and treated as in (b). Apoptosis was assessed in the population of transfected cells by evaluation of cPARP and nuclear morphology. ${ }^{*} P<0.05$, doxo versus control; ${ }^{* *} P<0.05$, Gli1 + doxo versus empty + doxo. (d) D283 medulloblastoma cells were transfected with siPCAF or siCTR and then treated with $2 \mu \mathrm{M}$ doxorubicin. Percent of BrdU incorporation was assessed as described in (b). ${ }^{\star} P<0.05$, doxo versus control; ${ }^{* *} P<0.05$ siPCAF + doxo versus siCTR + doxo. (e) D283 cells were transfected and treated as in (d). Apoptosis was evaluated as described in (c). ${ }^{*} P<0.05$, doxo versus control; ${ }^{* *} P<0.05$ siPCAF + doxo vs siCTR + doxo. (f) Daoy medulloblastoma cells were transfected with the specified siRNA and with the indicated plasmids. Percent of BrdU incorporation was assayed to monitor cell proliferation. ${ }^{*} P<0.05$, siGli1 versus siCTR or PCAF/siCTR versus empty/siCTR. (g) Daoy cells were transfected with the indicated plasmids, grown with G418 for 2 weeks, fixed and stained with crystal violet. Colony formation assay was performed to monitor cell proliferation. Counts from the colony formation assays and representative images from experiments are shown. ${ }^{*} P<0.05$, PCAF or Gli1 versus empty. (h) Cells derived from triplicate plates from the colony assays were grown in culture as monolayers, and cell growth rates were determined by analyzing cumulative cell number over time. Cells were counted in triplicate. Unless otherwise indicated, all experiments were performed in triplicate, and mean \pm S.D. is shown

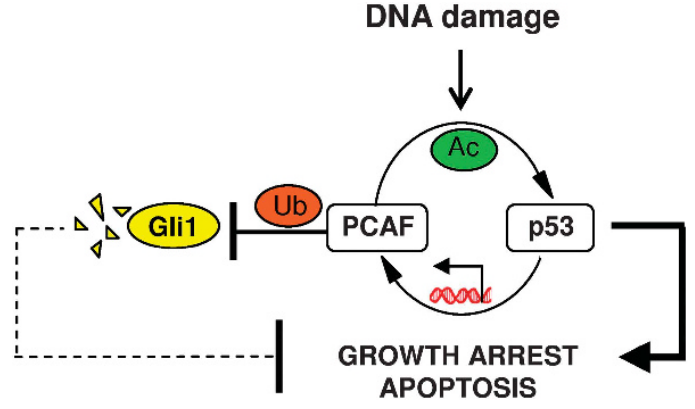

Figure 8 Model of p53/PCAF/Gli1 autoregulatory loop. In response to genotoxic stress, PCAF acetylates and enhances p53 function that in turn induces transcriptional activation of PCAF itself. The enhancement of PCAF levels leads to ubiquitination and degradation of Gli1, through PCAF intrinsic E3-ligase activity. The activation of the p53/PCAF/Gli1 circuitry releases cell cycle checkpoints (p53), thus preventing uncontrolled cell growth and promoting apoptosis. In contrast, inappropriate activation of $\mathrm{Hh} / \mathrm{Gli}$ or loss of DNA damaged-induced inhibitory signals (p53 and PCAF) determines uncontrolled cell proliferation, leading to tumorigenesis cells in MEM plus $20 \%$ and $10 \%$ FBS, respectively. Transient transfections were performed using Lipofectamine 2000 or Lipofectamine Plus (Invitrogen, Eugene, OR, USA). For RNA interference, D283 and MEF Ptch1 $1^{-1-}$ cells were transfected with scrambled or PCAF short interfering oligos (Sigma Aldrich, St. Louis, MO, USA) using Dharmafect 2 (Dharmacon, Lafayette, CO, USA); Daoy cells were transfected with scrambled or Gli1 short interfering oligos (Dharmacon) using Dharmafect 2. Cells were treated with SAG (Alexis Biochemicals, Farmingdale, NY, USA), MG132 (Calbiochem, Nottingham, UK), doxorubicin, cisplatin, cycloheximide (Sigma Aldrich).

Plasmids. pCX-PCAF, pCX-PCAF $\triangle \mathrm{HAT}$ (lacking aa 550-623) and pCl-p300 were a gift from M Fanciulli (Regina Elena Cancer Institute, Italy). PCAF $\Delta U b$ was constructed, as previously described, using PCR-amplified fragments of amino acids 1-121, and 242 to the stop codon, fused in frame with Flag epitope-tagged. ${ }^{33}$

Gli1 constructs and Gli-RE-Luc (containing 12 repeated Gli consensus sequences in a TK minimal promoter vector) have been described previously. ${ }^{35}$ Gli1 Dc was kindly provided by Oro A. ${ }^{11}$ Gli1 is described previously. ${ }^{36}$ Ubiquitin-Flag constructs were provided by I Dikic (Goethe University, Germany). Lentiviral vectors, pLV-WPXL-shp53, pLV-WPXL-shCTR, pLKO.1-puro-shCTR and 
pLKO.1-puro-shGli1 (kindly provided by Barbara Stecca, Istituto Toscano Tumori, Italy) were transfected into HEK293T packaging cells, and after $48 \mathrm{~h}$, viral supernatant was collected.

Antibodies. Mouse anti-Gli1 and rabbit anti- $\gamma \mathrm{H} 2 \mathrm{AX}$ were from Cell Signaling (Beverly, MA, USA); rabbit anti-Gli1, mouse anti-PCAF, mouse anti-Ub, mouse anti-p53, goat anti-Actin, mouse anti-p300, mouse anti-HA, anti-GFP-HRP, antiHA-HRP and HRP-conjugated secondary antibodies were purchased from Santa Cruz Biotechnology (Santa Cruz, CA, USA); rabbit anti-p53 was kindly provided by S Soddu (Regina Elena Cancer Institute, Italy); rabbit anti-acetylated lysine was from Upstate Biotechnology (Lake Placid, NY, USA); mouse anti-Flag-HRP was from Sigma Aldrich; 594- or 488-conjugated anti-mouse and anti-rabbit secondary antibodies were purchased from Molecular Probes (Invitrogen).

Biochemical methods. After transfection or drug treatments cells were lysed in Tris- $\mathrm{HCl}(\mathrm{pH} \mathrm{7.6)} 50 \mathrm{mM}, \mathrm{NaCl} 150 \mathrm{mM}, \mathrm{NP}-401 \%$, EDTA $5 \mathrm{mM}$, deoxycholic acid sodium salt $0.5 \%, \mathrm{NaF} 100 \mathrm{mM}$ and protease inhibitors. The lysates were centrifuged at $13000 \mathrm{~g}$ for $20 \mathrm{~min}$. For immunoblotting, protein extract was separated by SDS-PAGE. Immunoprecipitation, acetylation and in vivo ubiquitination assays were carried out as described previously. ${ }^{32,35}$ Briefly, for ubiquitination assay, HEK293T transfected with various plasmids or D283 cells treated with doxorubicin were lysed with denaturing buffer (1\% SDS, $50 \mathrm{mM}$ Tris at $\mathrm{pH} 7.5,0.5 \mathrm{mM}$ EDTA, $1 \mathrm{mM}$ DTT) to disrupt protein-protein interactions and then the lysates were diluted 10 times with lysis buffer and subjected to immunoprecipitation with antibodies (indicated in Figures 4-6 and Supplementary Figures 4 and 5) overnight at $4{ }^{\circ} \mathrm{C}$. The immunoprecipitates were then washed five times with the lysis buffer described above, resuspended in sample loading buffer, boiled for $5 \mathrm{~min}$, resolved in a 3-8\% SDS-PAGE (Invitrogen) and then subjected to immunoblot analysis. Polyubiquitinated species were detected using anti-HA, antiFlag or anti-Ub antibodies. For acetylation assay, HEK293T were transfected with the indicated plasmids and cell lysates were immunoprecipitated with anti-Gli1 (Santa Cruz). Acetylated species were detected using anti-acetylated lysine (Upstate Biotechnology).

GST-pulldown assay. GST recombinant proteins were bound to glutathione beads (GE Healthcare, Pittsburgh, PA, USA) and incubated for $1 \mathrm{~h}$ with in vitro translated protein in binding buffer (4-(2-hydroxyethyl)-1-piperazineethanesulfonic acid $20 \mathrm{mM}, \mathrm{MgCl} 22 \mathrm{mM}, \mathrm{KCl} 100 \mathrm{mM}, 20 \%$ glycerol, EDTA $0.2 \mathrm{mM}, 0.05 \%$ NP40) and analyzed by immunoblotting.

Luciferase, cell proliferation, colony formation and apoptosis assays. Luciferase and renilla activity were assayed with a dual-luciferase assay system (Promega, Madison, WI, USA). Results are expressed as luciferase/ renilla ratios and represent the mean \pm S.D. of at least three experiments, each performed in triplicate. Cell proliferation was evaluated by BrdU incorporation as previously described. ${ }^{45}$ Briefly, cells were treated with doxorubicin or transfected with the indicated plasmid. After the BrdU pulse, cells were fixed with $4 \%$ paraformaldehyde and permeabilized with $0.1 \%$ Triton X-100, and BrdU detection (Roche, Welwyn Garden City, UK) was performed according to the manufacturer's instructions. Nuclei were counterstained with Hoechst reagent. At least 500 nuclei were counted in triplicate, and the number of BrdU-positive nuclei was recorded. Apoptosis was measured by analysis of nuclear morphology and immunofluorescence using rabbit anti-cPARP antibody (Promega). Cells were fixed, permeabilized and counterstained as above described. At least 200 cells were counted in each well and experiments were conducted in triplicate. For colony formation assays, $1 \times 10^{4}$ transfected or transduced Daoy Mb cells were plated in $10-\mathrm{cm}$-diameter dishes, and after 2 weeks of neomycine or puromycin selection, cell colonies were counted after staining in $20 \%$ methanol and crystal violet. Cells derived from triplicate plates from the colony assays were grown in culture as monolayers, and cell growth rates were determined by analyzing cumulative cell numbers over time as previously described. ${ }^{46}$ Cells were counted in triplicate.

mRNA expression analysis. Total RNA was isolated with Trizol (Invitrogen) and reverse-transcribed with Superscript II reverse transcriptase and random hexamers (Invitrogen). Quantitative PCR (Q-PCR) analysis of Gli1, PCAF, p300, Bmi1, Hip1, CyclinD2, Bmp2 and Bcl2 mRNA expression was performed on each cDNA sample using the ABI Prism 7900 Sequence Detection System employing Assay-on-Demand Reagents (Applied Biosystems, Foster City, CA, USA). A reaction mixture containing CDNA template, TaqMan Universal PCR master mix ( $\mathrm{ABI}$ ) and primer probe mixture was amplified using standard Q-PCR thermal cycler parameters. Each amplification reaction was performed in triplicate and the average of the three threshold cycles was used to calculate the amount of transcript in the sample (using SDS version 1.7a software). mRNA quantification was expressed, in arbitrary units, as the ratio of the sample quantity to the quantity of the calibrator. All values were normalized with three endogenous controls, $\mathrm{GAPDH}, \beta$-actin and HPRT, which yielded similar results.

Statistical analysis. Statistical analysis was performed using StatView 4.1 software (Abacus Concepts, Berkeley, CA, USA). Statistical differences were analyzed with the Mann-Whitney U-test for non-parametric values and a $P<0.05$ was considered significant. Results are expressed as mean \pm S.D. from an appropriate number of experiments (at least three biological replicas), as indicated in the figure legends.

\section{Conflict of Interest}

The authors declare no conflict of interest.

Acknowledgements. We thank MP Scott for the gift of MEF Ptch $1^{-1-}$ cells, M Fanciulli for providing PCAF and PCAF $\triangle H A T$, A Oro for Gli1 Dc plasmid and B Stecca for lentiviral constructs; M Levrero for helpful suggestions; D Cucchi for experimental support. This work was supported by AIRC (Associazione Italiana Ricerca Cancro), AIRC 5XMILLE, MIUR FIRB and PRIN projects, Ministry of Health, EU HEALING grant no. 238186, IIT (Italian Institute of Technology), ASI (Agenzia Spaziale Italiana) and Pasteur Institute/Cenci Bolognetti Foundation.

1. Ruiz i, Altaba A. Hedgehog-Gli Signaling in Human Diseases. Plenum, 2006.

2. Barakat MT, Humke EW, Scott MP. Learning from Jekyll to control Hyde: Hedgehog signaling in development and cancer. Trends Mol Med 2010; 16: 337-348.

3. Teglund S, Toftgard R. Hedgehog beyond medulloblastoma and basal cell carcinoma. Biochim Biophys Acta 2010; 1805: 181-208.

4. Mabbott DJ, Spiegler BJ, Greenberg ML, Rutka JT, Hyder DJ, Bouffet E et al. Serial evaluation of academic and behavioral outcome after treatment with cranial radiation in childhood. J Clin Oncol 2005; 23: 2256-2263.

5. Mueller S, Chang S. Pediatric brain tumors: current treatment strategies and future therapeutic approaches. Neurotherapeutics 2009; 6: 570-586.

6. Thompson MC, Fuller C, Hogg TL, Dalton J, Finkelstein D, Lau CC et al. Genomics identifies medulloblastoma subgroups that are enriched for specific genetic alterations. J Clin Oncol 2006; 20: 1924-1931.

7. Ruppert JM, Vogelstein B, Kinzler KW. The zinc finger protein GLI transforms primary cells in cooperation with adenovirus E1A. Mol Cell Biol 1991; 11: 1724-1728.

8. Kimura H, Stephen D, Joyner A, Curran T. Gli1 is important for medulloblastoma formation in Ptc1 +/- mice. Oncogene 2005; 24: 4026-4036.

9. Ruiz i, Altaba A. Hedgehog signaling and the Gli code in stem cells, cancer, and metastases. Sci Signal 2011; 4: pt9.

10. Jiang J, Struhl G. Regulation of the Hedgehog and Wingless signaling pathways by the F-box/WD40-repeat protein Slimb. Nature 1998; 391: 493-496.

11. Huntzicker EG, Estay IS, Zhen H, Lokteva LA, Jackson PK, Oro AE et al. Dual degradation signals control Gli protein stability and tumor formation. Genes Dev 2006; 20: 276-281.

12. Di Marcotullio L, Ferretti E, Greco A, De Smaele E, Screpanti I, Gulino A et al. Multiple ubiquitin-dependent processing pathways regulate Hedgehog/Gli signaling. Cell Cycle 2007; 6: 390-393.

13. Di Marcotullio L, Canettieri G, Infante P, Greco A, Gulino A. Protected from the inside: endogenous histone deacetylase inhibitors and the road to cancer. Biochim Biophys Acta 2011; 1815: 241-252.

14. Jia J. Phosphorylation regulation of Hedgehog signaling. Vitam Horm 2012; 88: 253-272.

15. Gulino A, Di Marcotullio L, Canettieri G, De Smaele E, Screpanti I. Hedgehog/Gli control by ubiquitination/acetylation interplay. Vitam Horm 2012; 88: 211-227.

16. Stecca B, Ruiz i, Altaba A. A GLI1-p53 inhibitory loop controls neural stem cell and tumour cell numbers. EMBO J 2009; 28: 663-676.

17. Sakaguchi K, Herrera JE, Saito S, Miki T, Bustin M, Vassilev A et al. DNA damage activates p53 through a phosphorylation-acetylation cascade. Genes Dev 1998; 12: 2831-2841.

18. Vousden KH, Prives C. Blinded by the light: the growing complexity of p53. Cell 2009; 137: 413-431.

19. Cheng $Q$, Chen J. Mechanism of $p 53$ stabilization by ATM after DNA damage. Cell Cycle 2010; 9: 472-478.

20. Wetmore $C$, Eberhart DE, Curran T. Loss of $\mathrm{p} 53$ but not ARF accelerates medulloblastoma in mice heterozygous for patched. Cancer Res 2001; 61: 513-516.

21. Zindy F, Uziel T, Ayrault O, Calabrese C, Valentine M, Rehg JE et al. Genetic alterations in mouse medulloblastomas and generation of tumors de novo from primary cerebellar granule neuron precursors. Cancer Res 2007; 67: 2676-2684. 
22. Abe Y, Oda-Sato E, Tobiume K, Kawauchi K, Taya Y, Okamoto K et al. Hedgehog signaling overrides p53-mediated tumor suppression by activating Mdm2. Proc Natl Acad Sci USA 2008; 105: 4838-4843.

23. Malek R, Matta J, Taylor N, Perry ME, Mendrysa SM. The p53 inhibitor MDM2 facilitates Sonic Hedgehog-mediated tumorigenesis and influences cerebellar foliation. PLoS One 2011; 6: e17884.

24. Malkin D, Li FP, Strong LC, Fraumeni Jr JF, Nelson CE, Kim DH et al. Germ line p53 mutations in a familial syndrome of breast cancer, sarcomas, and other neoplasms. Science 1990; 250: 1233-1238.

25. Srivastava S, Zou ZQ, Pirollo K, Blattner W, Chang EH. Germ-line transmission of a mutated p53 gene in a cancer-prone family with Li-Fraumeni syndrome. Nature 1990; 348 747-749.

26. Taylor MD, Mainprize TG, Rutka JT. Molecular insight into medulloblastoma and centra nervous system primitive neuroectodermal tumor biology from hereditary syndromes: a review. Neurosurgery 2000; 47: 888-901.

27. Low JA, de Sauvage FJ. Clinical experience with Hedgehog pathway inhibitors. J Clin Oncol 2010; 28: 5321-5326.

28. Rausch T, Jones DT, Zapatka M, Stütz AM, Zichner T, Weischenfeldt J et al. Genome sequencing of pediatric medulloblastoma links catastrophic DNA rearrangements with TP53 mutations. Cell 2012; 148: 59-71.

29. Sasaki H, Hui C, Nakafuku M, Kondoh H. A binding site for Gli proteins is essential for HNF. 3beta floor plate enhancer activity in transgenics and can respond to Shh in vitro. Development 1997; 124: 1313-1322.

30. Saylors RLI, Sidransky D, Friedman HS, Bigner SH, Bigner DD, Vogelstein B et al. Infrequent p53 gene mutations in medulloblastoma. Cancer Res 1991; 51: 4721-4723.

31. Watts GS, Oshiro MM, Junk DJ, Wozniak RJ, Watterson S, Domann FE et al. The acetyltransferase p300/CBP-associated factor is a p53 target gene in breast tumor cells. Neoplasia 2004; 6: 187-194.

32. Canettieri G, Di Marcotullio L, Greco A, Coni S, Antonucci L, Infante $P$ et al. Histone deacetylase and Cullin3-REN(KCTD11) ubiquitin ligase interplay regulates Hedgehog signalling through Gli acetylation. Nat Cell Biol 2010; 12 132-142.

33. Linares LK, Kiernan R, Triboulet R, Chable-Bessia C, Latreille D, Cuvier O et al. Intrinsic ubiquitination activity of PCAF controls the stability of the oncoprotein Hdm2. Nat Cell Biol 2007; 9: 331-338.
34. Grossman SR, Deato ME, Brignone C, Chan HM, Kung AL, Tagami $\mathrm{H}$ et al. Polyubiquitination of $p 53$ by a ubiquitin ligase activity of p300. Science 2003; 11: 342-344.

35. Di Marcotullio L, Ferretti E, Greco A, De Smaele E, Po A, Sico MA et al. Numb is a suppressor of Hedgehog signalling and targets Gli1 for Itch-dependent ubiquitination. Nat Cell Biol 2006; 8: 1415-1423.

36. Di Marcotullio L, Greco A, Mazzà D, Canettieri G, Pietrosanti L, Infante $P$ et al. Numb activates the E3 ligase Itch to control Gli1 function through a novel degradation signal. Oncogene 2011; 30: 65-76.

37. Lauth M, Bergstrom A, Shimokawa T, Toftgard R. Inhibition of GLI-mediated transcription and tumor cell growth by small-molecule antagonists. Proc Natl Acad Sci USA 2007; 104: $8455-8460$.

38. Mazumdar T, DeVecchio J, Agyeman A, Shi T, Houghton JA. Blocking Hedgehog survival signaling at the level of the GLI genes induces DNA damage and extensive cell death in human colon carcinoma cells. Cancer Res 2011; 71: 5904-5914.

39. Ng JM, Curran T. The Hedgehog's tale: developing strategies for targeting cancer. Nature Rev Cancer 2011; 11: 493-501.

40. Rudin CM, Hann CL, Laterra J, Yauch RL, Callahan CA, Fu L et al. Treatment of medulloblastoma with hedgehog pathway inhibitor GDC-0449. N Engl J Med 2009; 361: 1173-1178.

41. Metcalfe C, de Sauvage FJ. Hedgehog fights back: mechanisms of acquired resistance against Smoothened antagonists. Cancer Res 2011; 71: 5057-5061.

42. Lane DP, Cheok CF, Lain S. p53-based cancer therapy. Cold Spring Harb Perspect Biol 2010; 2: a001222.

43. Ghassemifar S, Mendrysa SM. MDM2 antagonism by nutlin-3 induces death in human medulloblastoma cells. Neurosci Lett 2012; 513: 106-110

44. Künkele A, De Preter K, Heukamp L, Thor T, Pajtler KW, Hartmann W et al. Pharmacological activation of the p53 pathway by nutlin-3 exerts anti-tumoral effects in medulloblastomas. NeuroOncol 2012; 14: 859-869.

45. De Smaele E, Di Marcotullio L, Moretti M, Pelloni M, Occhione MA, Infante P et al. Identification and characterization of $\mathrm{KCASH} 2$ and $\mathrm{KCASH} 3$, novel Cullin3 adaptors suppressing histone deacetylase and Hedgehog activity in medulloblastoma. Neoplasia 2011; 13: 374-385.

46. Canettieri G, Coni S, Della Guardia M, Nocerino V, Antonucci L, Di Magno L et al. The coactivator CRTC1 promotes cell proliferation and transformation via AP-1. Proc Natl Acad Sci USA 2009; 106: 1445-1450.

\section{Supplementary Information accompanies this paper on Cell Death and Differentiation website (http://www.nature.com/cdd)}

